\title{
Influence of Relapse in a Giving Up Smoking Model
}

\author{
Hai-Feng Huo and Cheng-Cheng Zhu \\ Department of Applied Mathematics, Lanzhou University of Technology, Lanzhou, Gansu 730050, China \\ Correspondence should be addressed to Hai-Feng Huo; huohf70@sohu.com
}

Received 8 November 2012; Revised 7 December 2012; Accepted 20 December 2012

Academic Editor: Sanyi Tang

Copyright (C) 2013 H.-F. Huo and C.-C. Zhu. This is an open access article distributed under the Creative Commons Attribution License, which permits unrestricted use, distribution, and reproduction in any medium, provided the original work is properly cited.

Smoking subject is an interesting area to study. The aim of this paper is to derive and analyze a model taking into account light smokers compartment, recovery compartment, and two relapses in the giving up smoking model. Stability of the model is obtained. Some numerical simulations are also provided to illustrate our analytical results and to show the effect of controlling the rate of relapse on the giving up smoking model.

\section{Introduction}

As early as 1889 , people have established a model for the spread of infectious diseases. Then the spread rule and trend of the model were studied by analying the stability of the solutions ([1-4] and the references cited therein). De la Sen and Alonso-Quesada [3] present several simple linear vaccination-based control strategies for a SEIR propagation disease model and study the stability of this model. De La Sen et al. [4] discuss a generalized time-varying SEIR propagation disease model subject to delays which potentially involves mixed regular and impulsive vaccination rules, and in this paper the authors were using the good methods to study the dynamic behavior of the model especially the positivity of the model. There are many methods to discuss the stability, one of the most powerful techniques for qualitative analysis of a dynamical system is Direct Lyapunov Method [5]. This method employs an appropriate auxiliary function, called a Lyapunov function. For example, [6-8] use this method to discuss the stability of the model. In addition, there are a number of articles which use Routh-Hurwitz theory to explore the stability, see for example $[9,10]$.

In recent years, many types of epidemic models are discussed, such as virus dynamics models [11, 12], tuberculosis models $[13,14]$, and HIV models $[15,16]$.

Due to the increasing in the number of smokers, tobacco use is also as a disease to be treated. In order to explore the spread rule of smoking, quit smoking model is developed.
Castillo-Garsow et al. [17] proposed a simple mathematical model for giving up smoking in the first time. In this model, a total constant population was divided into three classes: potential smokers, that is, people who do not smoke yet but might become smokers in the future $(P)$, smokers $(S)$, and quit smokers $(Q)$. Zaman [18] extended the work of CastilloGarsow et al. [17] by adding the population of occasional smokers in the model, and presented qualitative behavior of the model. Zaman [19] presented the optimal campaigns in the smoking dynamics. They consider two possible control variables in the form of education and treatment campaigns oriented to decrease the attitude towards smoking and first showed the existence of an optimal control for the control problem.

However, in real life, the usual quit smokers are only temporary quit smokers. Some of them may relapse since they contact with smokers again, and the others may become permanent quit smokers. Statistics also show that $15 \%$ quit smokers may relapse when they contact with smokers. Enlightening by the previously mentioned cases, we present a model, which extend the models in [17-19] by taking into account the temporary quit smoker compartment $(R)$ and two kinds of relapses, that is, once a smoker temporary quits smoking he/she may become a light or occasion smoker or a persistent smoker again. First, we derive the basic reproductive number, and discuss the positivity of the solution for the giving up smoking model. Then, we analyze the stability of equilibria by Lyapunov Method and Routh-Hurwitz theory. 
Finally, by estimating of parameter, we present the numerical simulation. Moreover, the numerical simulation shows that we can greatly improve the effect of quit smoking by using some methods, such as treatment and education, controlling the rate of relapse.

The organization of this paper is as follow: the model is given under some assumption in Section 2. The basic reproductive number, existence, and the stability of equilibria are investigated in Section 3. Some numerical simulations are given in Section 4. The paper ends with a discussion in Section 5 .

\section{The Model Formulation}

2.1. System Description. In this paper, we establish the giving up smoking model as Figure 1. Table 1 presents the parameters description of the model.

From Figure 1, the total population is divided into five compartments, namely, the potential smokers compartment $(P)$, light or occasion smokers compartment $(L)$, persistent smokers compartment $(S)$, temporary quit smokers $(R)$ that is the people who did some efforts to stop smoking, and quit smokers forever group $(Q)$. As we know that if you smoke more, the harm of nicotine on the body will be greater. So the death rate is also higher. Hence, we can further assume that $d_{2}<d_{3}$. The total population size is $N(t)$, where

$$
N(t)=P(t)+L(t)+S(t)+R(t)+Q(t) .
$$

The transfer diagram leads to the following system of ordinary differential equations:

$$
\begin{gathered}
\frac{d P(t)}{d t}=b-\beta_{1} P(t) L(t)-\left(d_{1}+\mu\right) P(t), \\
\frac{d L(t)}{d t}=\beta_{1} P(t) L(t)-\beta_{2} L(t) S(t)+\rho_{2} L(t) R(t) \\
-\left(d_{2}+\mu\right) L(t), \\
\frac{d S(t)}{d t}=\beta_{2} L(t) S(t)+\rho_{1} S(t) R(t)-\left(\omega+d_{3}+\mu\right) S(t), \\
\frac{d R(t)}{d t}=\omega S(t)-\rho_{1} S(t) R(t)-\rho_{2} L(t) R(t) \\
-\left(\gamma+d_{4}+\mu\right) R(t), \\
\frac{d Q(t)}{d t}=\gamma R(t)-\left(d_{5}+\mu\right) Q(t) .
\end{gathered}
$$

2.2. Positivity and Boundedness of Solutions. For system (2), to ensure that the solutions of the system with positive initial conditions remain positive for all $t>0$, it is necessary to prove that all the state variables are nonnegative. Similar to the proof of $[3,4,13]$, we have the following lemma.

Lemma 1. If $P(0)>0, L(0)>0, S(0)>0, R(0)>0, Q(0)>$ 0 , the solutions $P(t), L(t), S(t), R(t), Q(t)$ of system (2) are positive for all $t>0$.
Proof. If the conclusion does not hold, then at least one of $P(t), L(t), S(t), R(t), Q(t)$ is not positive. Thus, we have one of the following five cases.

(1) There exists a first time $t_{1}$ such that

$$
\begin{gathered}
P\left(t_{1}\right)=0, \quad P^{\prime}\left(t_{1}\right)<0, \quad L(t) \geq 0, \quad S(t) \geq 0, \\
R(t) \geq 0, \quad Q(t) \geq 0, \quad 0 \leq t \leq t_{1} .
\end{gathered}
$$

(2) There exists a first time $t_{2}$ such that

$$
\begin{gathered}
L\left(t_{2}\right)=0, \quad L^{\prime}\left(t_{2}\right)<0, \quad P(t) \geq 0, \quad S(t) \geq 0, \\
R(t) \geq 0, \quad Q(t) \geq 0, \quad 0 \leq t \leq t_{2} .
\end{gathered}
$$

(3) There exists a first time $t_{3}$ such that

$$
\begin{gathered}
S\left(t_{3}\right)=0, \quad S^{\prime}\left(t_{3}\right)<0, \quad P(t) \geq 0, \quad L(t) \geq 0, \\
R(t) \geq 0, \quad Q(t) \geq 0, \quad 0 \leq t \leq t_{3} .
\end{gathered}
$$

(4) There exists a first time $t_{4}$ such that

$$
\begin{gathered}
R\left(t_{4}\right)=0, \quad R^{\prime}\left(t_{4}\right)<0, \quad P(t) \geq 0, \quad L(t) \geq 0, \\
S(t) \geq 0, \quad Q(t) \geq 0, \quad 0 \leq t \leq t_{4} .
\end{gathered}
$$

(5) There exists a first time $t_{5}$ such that

$$
\begin{gathered}
Q\left(t_{5}\right)=0, \quad Q^{\prime}\left(t_{5}\right)<0, \quad P(t) \geq 0, \quad L(t) \geq 0, \\
S(t) \geq 0, \quad R(t) \geq 0, \quad 0 \leq t \leq t_{5} .
\end{gathered}
$$

In case (1), we have

$$
P^{\prime}\left(t_{1}\right)=b>0,
$$

which is a contradiction to $P^{\prime}\left(t_{1}\right)<0$.

In case (2), we have

$$
L^{\prime}\left(t_{2}\right)=0 \text {, }
$$

which is a contradiction to $L^{\prime}\left(t_{2}\right)<0$.

In case (3), we have

$$
S^{\prime}\left(t_{3}\right)=0,
$$

which is a contradiction to $S^{\prime}\left(t_{3}\right)<0$.

In case (4), we have

$$
R^{\prime}\left(t_{4}\right)=\omega S\left(t_{4}\right)>0,
$$

which is a contradiction to $R^{\prime}\left(t_{4}\right)<0$.

In case (5), we have

$$
Q^{\prime}\left(t_{5}\right)=\gamma R\left(t_{5}\right)>0,
$$

which is a contradiction to $Q^{\prime}\left(t_{5}\right)<0$.

Thus, the solutions $P(t), L(t), S(t), R(t), Q(t)$ of system (2) remain positive for all $t>0$. 


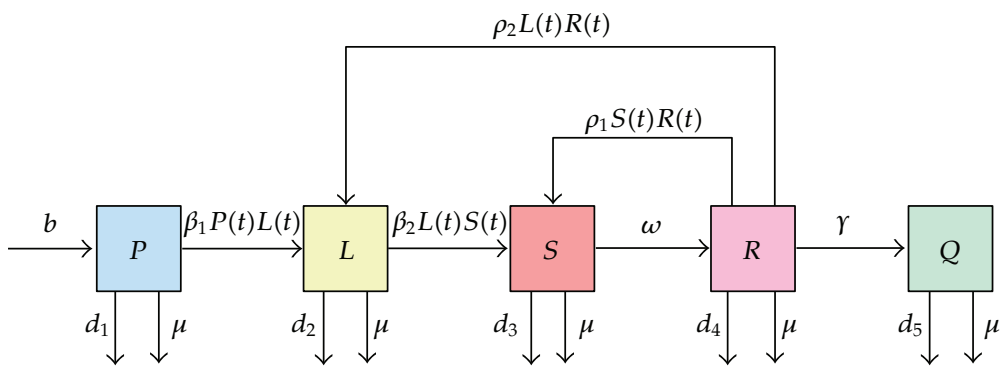

FIgURE 1: Transfer diagram for the dynamics of giving up smoking model.

TABLE 1: The parameters description of giving up smoking model.

\begin{tabular}{ll}
\hline Parameter & Description \\
\hline$b$ & $\begin{array}{l}\text { The total recruitment number into this homogeneous social mixing community. } \\
\text { Transmission coefficient from the potential smokers compartment to the light or occasion smokers } \\
\beta_{1}\end{array}$ \\
$\beta_{2}$ & $\begin{array}{l}\text { Transmission coefficient from the light or occasion smokers compartment to the persistent smokers } \\
\text { compartment. }\end{array}$ \\
$\rho_{1}$ & The relapse rate of which temporary quit people contact with persistent smokers. \\
$\rho_{2}$ & The relapse rate of which temporary quit people contact with light smokers. \\
$\omega$ & The temporary quit smoking rate. \\
$\gamma$ & The permanent quit smoking rate. \\
$\mu$ & Naturally death rate. \\
$d_{i}, i=1, \ldots, 5$ & The smoking-related death rate.
\end{tabular}

Lemma 2. All feasible solution of the system (2) are bounded and enter the region

$$
\Omega=\left\{(P, L, S, R, Q) \in R_{+}^{5}: P+L+S+R+Q \leq \frac{b}{\mu}\right\} .
$$

Proof. Let $(P, L, S, R, Q) \in R_{+}^{5}$ be any solution with nonnegative initial condition: adding the first four equations of (2), we have

$$
\begin{aligned}
\frac{d}{d t}(P+ & L+S+R+Q) \\
= & b-\left(d_{1}+\mu\right) P-\left(d_{2}+\mu\right) L-\left(d_{3}+\mu\right) S \\
& -\left(d_{4}+\mu\right) R-\left(d_{5}+\mu\right) Q \\
= & b-\mu(P+L+S+R+Q) \\
& -\left(d_{1} P+d_{2} L+d_{3} S+d_{4} R+d_{5} Q\right) \\
\leq & b-\mu N .
\end{aligned}
$$

It follows that

$$
0 \leq N(t) \leq \frac{b}{\mu}+N(0) e^{-\mu t},
$$

where $N(0)$ represents initial values of the total population. Thus $0 \leq N(t) \leq b / \mu$, as $t \rightarrow \infty$. Therefore all feasible solutions of system (2) enter the region

$$
\Omega=\left\{(P, L, S, R, Q) \in R_{+}^{5}: P+L+S+R+Q \leq \frac{b}{\mu}\right\} .
$$

Hence, $\Omega$ is positively invariant, and it is sufficient to consider solutions of system (2) in $\Omega$. Existence, uniqueness, and continuation results of system (2) hold in this region. It can be shown that $N(t)$ is bounded and all the solutions starting in $\Omega$ approach enter or stay in $\Omega$.

\section{Analysis of the Model}

In this section, we will analyze the existence of equilibria of system (2).

3.1. The Existence of Equilibria and the Basic Reproduction Number. The model (2) has a smoking-free equilibrium given by (see Theorem 3 (1))

$$
E_{0}=\left(\frac{b}{d_{1}+\mu}, 0,0,0,0\right)
$$

In the following, the basic reproduction number of system (2) will be obtained by the next generation matrix method formulated in [21].

Let $x=(L, S, R, Q, P)^{T}$; then system (2) can be written as

$$
\frac{d x}{d t}=\mathscr{F}(x)-\mathscr{V}(x)
$$


where

$$
\begin{gathered}
\mathscr{F}(x)=\left(\begin{array}{c}
\beta_{1} P L \\
0 \\
0 \\
0 \\
0
\end{array}\right), \\
\mathscr{V}(x)=\left(\begin{array}{c}
\beta_{2} L S+\left(d_{2}+\mu\right) L-\rho_{2} L R \\
-\beta_{2} L S-\rho_{1} S R+\left(\omega+d_{3}+\mu\right) S \\
-\omega S+\rho_{1} S R+\rho_{2} L R+\left(\gamma+d_{4}+\mu\right) R \\
-\gamma R+\left(d_{5}+\mu\right) Q \\
-b+\beta_{1} P L+\left(d_{1}+\mu\right) P
\end{array}\right) .
\end{gathered}
$$

The Jacobian matrices of $\mathscr{F}(x)$ and $\mathscr{V}(x)$ at the smoking-free equilibrium $E_{0}$ are, respectively,

$$
\begin{aligned}
& D \mathscr{F}\left(E_{0}\right)=\left(\begin{array}{cc}
F_{4 \times 4} & 0 \\
0 & 0
\end{array}\right),
\end{aligned}
$$

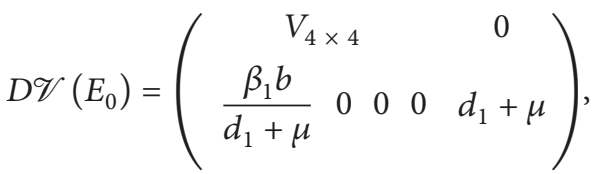

where

$$
\begin{gathered}
F_{4 \times 4}=\left(\begin{array}{cccc}
\frac{\beta_{1} b}{d_{1}+\mu} & 0 & 0 & 0 \\
0 & 0 & 0 & 0 \\
0 & 0 & 0 & 0 \\
0 & 0 & 0 & 0
\end{array}\right), \\
V_{4 \times 4}=\left(\begin{array}{cccc}
d_{2}+\mu & 0 & 0 & 0 \\
0 & \omega+d_{3}+\mu & 0 & 0 \\
0 & -\omega & \gamma+d_{4}+\mu & 0 \\
0 & 0 & -\gamma & d_{5}+\mu
\end{array}\right) .
\end{gathered}
$$

In order to simplify the calculation, letting $b_{1}=d_{2}+\mu, b_{2}=$ $\omega+d_{3}+\mu, b_{3}=\gamma+d_{4}+\mu, b_{4}=d_{5}+\mu$, we obtain

$$
V^{-1}=\left(\begin{array}{cccc}
\frac{1}{b_{1}} & 0 & 0 & 0 \\
0 & \frac{1}{b_{2}} & 0 & 0 \\
0 & \frac{\omega}{\left(\omega+b_{2}\right)\left(\gamma+b_{3}\right)} & \frac{1}{\gamma+b_{3}} & 0 \\
0 & \frac{\omega \gamma}{\left(\omega+b_{2}\right)\left(\gamma+b_{3}\right) b_{4}} & \frac{\gamma}{\left(\gamma+b_{3}\right) b_{4}} & \frac{1}{b_{4}}
\end{array}\right) .
$$

The basic reproduction number, denoted by $R_{0}$, is thus given by

$$
R_{0}=\rho\left(F V^{-1}\right)=\frac{\beta_{1} b}{\left(d_{1}+\mu\right) b_{1}}=\frac{\beta_{1} b}{\left(d_{1}+\mu\right)\left(d_{2}+\mu\right)} .
$$

Throughout this paper, we denote

$$
\alpha_{3}=\left(\omega+d_{3}+\mu\right), \quad \alpha_{4}=\left(\gamma+d_{4}+\mu\right) .
$$

Theorem 3. For the giving up smoking model (2), there exist the following three types of equilibrium.

(1) For all parameter values, system (2) exists the smokingfree equilibrium $E_{0}\left(b /\left(d_{1}+\mu\right), 0,0,0,0\right)$.

(2) If $R_{0}>1$, there exists the occasion smoking equilibrium $E_{L}\left(\left(d_{2}+\mu\right) / \beta_{1}, b /\left(d_{2}+\mu\right)-\left(d_{1}+\mu\right) / \beta_{1}, 0,0,0\right)$, and there exists no occasion smoking equilibrium if $R_{0} \leq 1$.

(3) If $R_{2}=1 / R_{0}+\left(d_{2}+\mu\right) \alpha_{3} / b \beta_{2}<1, d_{3} \rho_{2}-d_{2}<$ 0 and $\rho_{2}>\max \left\{d_{2} \rho_{1} \omega / d_{3} \alpha_{3}, \rho_{1} \omega / \alpha_{3}\right\}$, then system (2) has positive smoking-present equilibrium $E^{*}\left(P^{*}, L^{*}, S^{*}, R^{*}, Q^{*}\right)$ where $R^{*} \in\left(0, \omega / \rho_{1}\right)$.

Moveover, $E^{*}\left(P^{*}, L^{*}, S^{*}, R^{*}, Q^{*}\right)$ satisfies the following equality:

$$
\begin{gathered}
L^{*}=\frac{\alpha_{3}-\rho_{1} R^{*}}{\beta_{2}}, \\
S^{*}=\frac{\rho_{1} \rho_{2} R^{*^{2}}-\alpha_{4} \beta_{2} R^{*}-\alpha_{3} \rho_{2} R^{*}}{\beta_{2}\left(\rho_{1} R^{*}-\omega\right)}, \\
P^{*}=\frac{1}{\beta_{1}} \frac{-\alpha_{4} \beta_{2} R^{*}-\left(d_{3}+\mu\right) \rho_{2} R^{*}+\left(\rho_{1} R^{*}-\omega\right)\left(d_{2}+\mu\right)}{\left(\rho_{1} R^{*}-\omega\right)}, \\
Q^{*}=\frac{\gamma}{d_{5}+\mu} R^{*},
\end{gathered}
$$

where $R^{*}$ is a positive solution of $f(R)=0$, where

$$
\begin{aligned}
f(R)= & \left(d_{1}+\mu\right) \frac{\rho_{1} \rho_{2} R^{2}-\alpha_{4} \beta_{2} R-\alpha_{3} \rho_{2} R}{\beta_{1}\left(\rho_{1} R-\omega\right)} \\
& -\left(d_{1}+\mu\right) \frac{\rho_{2}}{\beta_{1}} R+\frac{\left(d_{1}+\mu\right)\left(d_{2}+\mu\right)}{\beta_{1}} \\
& +\left(d_{2}+\mu\right) \frac{\alpha_{3}-\rho_{1 R}}{\beta_{2}} \\
& +\left(d_{3}+\mu\right) \frac{\rho_{1} \rho_{2} R^{2}-\alpha_{4} \beta_{2} R-\alpha_{3} \rho_{2} R}{\beta_{2}\left(\rho_{1} R-\omega\right)}+\left(d_{4}+\mu\right) R \\
& +\gamma R-b .
\end{aligned}
$$

Proof. It follows from system (2) that

$$
\begin{gathered}
b-\beta_{1} P(t) L(t)-\left(d_{1}+\mu\right) P(t)=0, \\
\beta_{1} P(t) L(t)-\beta_{2} L(t) S(t)+\rho_{2} L(t) R(t) \\
-\left(d_{2}+\mu\right) L(t)=0, \\
\beta_{2} L(t) S(t)+\rho_{1} S(t) R(t)-\left(\omega+d_{3}+\mu\right) S(t)=0, \\
\omega S(t)-\rho_{1} S(t) R(t)-\rho_{2} L(t) R(t) \\
-\left(\gamma+d_{4}+\mu\right) R(t)=0, \\
\gamma R(t)-\left(d_{5}+\mu\right) Q(t)=0 .
\end{gathered}
$$


(1) Letting $L=S=R=Q=0$ in (27), we can obtain the smoking-free equilibrium $E_{0}\left(b /\left(d_{1}+\mu\right), 0,0,0,0\right)$.

(2) If $R_{0}>1$, letting $S=R=Q=0$ in (27), we can obtain the occasion smoking equilibrium $E_{L}\left(\left(d_{2}+\right.\right.$ $\left.\mu) / \beta_{1}, b /\left(d_{2}+\mu\right)-\left(d_{1}+\mu\right) / \beta_{1}, 0,0,0\right)$.

(3) From third equation of the system (27), we obtain

$$
L=\frac{\alpha_{3}-\rho_{1} R}{\beta_{2}} \text {. }
$$

By adding the third equation and the fourth equation, we get

$$
\begin{aligned}
& \beta_{2} L(t) S(t)-\left(\omega+d_{3}+\mu\right) S(t)+\omega S(t) \\
& \quad-\left(\gamma+d_{4}+\mu\right) R(t)-\rho_{2} L(t) R(t)=0 .
\end{aligned}
$$

From this we have

$$
S=\frac{\rho_{1} \rho_{2} R^{2}-\alpha_{4} \beta_{2} R-\alpha_{3} \rho_{2} R}{\beta_{2}\left(\rho_{1} R-\omega\right)} .
$$

From the second equation of the system (27) we get

$$
\beta_{1} P(t)=\beta_{2} S(t)-\rho_{2} R(t)+\left(d_{2}+\mu\right) .
$$

Substituting $S$ into (31), we get

$$
\begin{aligned}
P & =\frac{\rho_{1} \rho_{2} R^{2}-\alpha_{4} \beta_{2} R-\alpha_{3} \rho_{2} R}{\beta_{1}\left(\rho_{1} R-\omega\right)}-\frac{\rho_{2}}{\beta_{1}} R+\frac{d_{2}+\mu}{\beta_{1}} \\
& =\frac{1}{\beta_{1}} \frac{-\alpha_{4} \beta_{2} R-\left(d_{3}+\mu\right) \rho_{2} R+\left(\rho_{1} R-\omega\right)\left(d_{2}+\mu\right)}{\left(\rho_{1} R-\omega\right)} .
\end{aligned}
$$

It follows from the fifth equation that

$$
Q=\frac{\gamma}{d_{5}+\mu} R
$$

Let

$$
\begin{aligned}
f(R)= & \left(d_{1}+\mu\right) P+\left(d_{2}+\mu\right) L+\left(d_{3}+\mu\right) S \\
& +\left(d_{4}+\mu\right) R+\left(d_{5}+\mu\right) Q-b \\
= & \left(d_{1}+\mu\right) \frac{\rho_{1} \rho_{2} R^{2}-\alpha_{4} \beta_{2} R-\alpha_{3} \rho_{2} R}{\beta_{1}\left(\rho_{1} R-\omega\right)} \\
& -\left(d_{1}+\mu\right) \frac{\rho_{2}}{\beta_{1}} R+\frac{\left(d_{1}+\mu\right)\left(d_{2}+\mu\right)}{\beta_{1}} \\
& +\left(d_{2}+\mu\right) \frac{\alpha_{3}-\rho_{1 R}}{\beta_{2}} \\
& +\left(d_{3}+\mu\right) \frac{\rho_{1} \rho_{2} R^{2}-\alpha_{4} \beta_{2} R-\alpha_{3} \rho_{2} R}{\beta_{2}\left(\rho_{1} R-\omega\right)} \\
& +\left(d_{4}+\mu\right) R+\gamma R-b .
\end{aligned}
$$

From (28) we can see that if $R<\alpha_{3} / \rho_{1}$, then $L>0$. From (30) we can see that if $R<\omega / \rho_{1}$, then $\rho_{1} R-\omega<0, \rho_{1} \rho_{2} R-\alpha_{4} \beta_{2}-$ $\alpha_{3} \rho_{2}<0$; these show that $S>0$. From (32), we can see that if $R<\omega / \rho_{1}$, then $P>0$. Therefore, if $R<\omega / \rho_{1}, P>0, L>0$, $S>0, Q>0$. In the following, we prove that the existence of positive solutions of $f(R)=0$. From (34), we know

$$
\lim _{R \rightarrow \omega / \rho_{1}^{-}} f(R)=+\infty .
$$

If $\left(d_{1}+\mu\right)\left(d_{2}+\mu\right) / \beta_{1}+\left(d_{2}+\mu\right) \alpha_{3} / \beta_{2}<b$, then

$$
f(0)=\frac{\left(d_{1}+\mu\right)\left(d_{2}+\mu\right)}{\beta_{1}}+\frac{\left(d_{2}+\mu\right) \alpha_{3}}{\beta_{2}}-b<0,
$$

$$
\begin{aligned}
f^{\prime}(R)= & \frac{\left(d_{1}+\mu\right)}{\beta_{1}} \frac{g(R)}{\left(\rho_{1} R-\omega\right)^{2}}-\left(d_{1}+\mu\right) \frac{\rho_{2}}{\beta_{1}}-\left(d_{2}+\mu\right) \frac{\rho_{1}}{\beta_{2}} \\
& +\frac{\left(d_{3}+\mu\right)}{\beta_{2}} \frac{g(R)}{\left(\rho_{1} R-\omega\right)^{2}}+\left(d_{4}+\mu\right)+\gamma,
\end{aligned}
$$

where

$$
g(R)=\rho_{1}^{2} \rho_{2} R^{2}-2 \rho_{1} \rho_{2} \omega R+\alpha_{4} \beta_{2} \omega+\alpha_{3} \rho_{2} \omega .
$$

Clearly, $R=\omega / \rho_{1}$ is the minimum point of $g(R)$. For $R \in$ $\left(0, \omega / \rho_{1}\right)$, we have

$$
g(R) \geq g\left(\frac{\omega}{\rho_{1}}\right)=\left(-\rho_{2} \omega+\alpha_{4} \beta_{2}+\alpha_{3} \rho_{2}\right) \omega>0 .
$$

Hence,

$$
\begin{aligned}
f^{\prime}(R)= & \frac{\left(d_{1}+\mu\right)}{\beta_{1}} \frac{\alpha_{4} \beta_{2} \omega+\alpha_{3} \rho_{2} \omega-\rho_{2} \omega^{2}}{\left(\rho_{1} R-\omega\right)^{2}} \\
& +\frac{h(R)}{\beta_{2}\left(\rho_{1} R-\omega\right)^{2}}+\left(d_{4}+\mu\right)+\gamma,
\end{aligned}
$$

where

$$
\begin{aligned}
h(R)= & d_{3} \rho_{1}^{2} \rho_{2} R^{2}-2 d_{3} \rho_{1} \rho_{2} \omega R+d_{3} \omega \alpha_{4} \beta_{2}+d_{3} \omega \alpha_{3} \rho_{2} \\
& +\mu \rho_{1}^{2} \rho_{2} R^{2}-2 \mu \rho_{1} \rho_{2} \omega R+\mu \omega \alpha_{4} \beta_{2}+\mu \omega \alpha_{3} \rho_{2} \\
& -d_{2} \rho_{1}^{3} R^{2}+2 d_{2} \rho_{1}^{2} \omega R-d_{2} \rho_{1} \omega^{2} \\
& -\mu \rho_{1}^{3} R^{2}+2 \mu \rho_{1}^{2} \omega R-\mu \rho_{1} \omega^{2} .
\end{aligned}
$$

Then

$$
\begin{aligned}
h^{\prime}(R)= & 2 d_{3} \rho_{1} \rho_{2}\left(\rho_{1} R-\omega\right)+2 \mu \rho_{1} \rho_{2}\left(\rho_{1} R-\omega\right) \\
& -2 d_{2} \rho_{1}\left(\rho_{1} R-\omega\right)-2 \mu\left(\rho_{1} R-\omega\right) \\
= & 2 \rho_{1}\left(\rho_{1} R-\omega\right)\left(d_{3} \rho_{2}-d_{2}\right) \\
& +2 \mu \rho_{1}\left(\rho_{1} R-\omega\right)\left(\rho_{2}-1\right),
\end{aligned}
$$

as we know that $\rho_{2}<1$ and $\rho_{1} R-\omega<0$. If $d_{3} \rho_{2}-d_{2}<0$, then for any $R \in\left(0, \omega / \rho_{1}\right)$, we have $h^{\prime}(R)>0$, so $h(R)$ is a strictly 
monotone increasing function on $\left(0, \omega / \rho_{1}\right)$. As we know that $\rho_{2}>\max \left\{d_{2} \rho_{1} \omega / d_{3} \alpha_{3}, \rho_{1} \omega / \alpha_{3}\right\}, d_{3}>d_{2}$ and $\alpha_{3}>\omega$, so

$$
\begin{aligned}
h(0)= & d_{3} \omega \alpha_{4} \beta_{2}+d_{3} \omega \alpha_{3} \rho_{2}+\mu \omega \alpha_{4} \beta_{2} \\
& +\mu \omega \alpha_{3} \rho_{2}-d_{2} \rho_{1} \omega^{2}-\mu \rho_{1} \omega^{2} \\
= & d_{3} \omega \alpha_{4} \beta_{2}+\mu \omega \alpha_{4} \beta_{2}+\omega\left(d_{3} \alpha_{3} \rho_{2}-d_{2} \rho_{1} \omega\right) \\
& \times \mu \omega\left(\alpha_{3} \rho_{2}-\rho_{1} \omega\right)>0 .
\end{aligned}
$$

Hence, $h(R)>0$ for any $R \in\left(0, \omega / \rho_{1}\right)$, so $f^{\prime}(R)>0$; that is, $f(R)$ is a strictly monotone increasing function on $\left(0, \omega / \rho_{1}\right)$. Therefore, $f(R)=0$ has an unique positive solutions on $\left(0, \omega / \rho_{1}\right)$. The proof is completed.

3.2. Qualitative Analysis. In this part we will discuss the qualitative behavior of the giving up smoking model (2).

\subsubsection{Stability of the Smoking-Free Equilibrium.}

Theorem 4. If $R_{0} \leq 1$, the smoking-free equilibrium $E_{0}$ is globally asymptotically stable.

Proof. We introduce the following Lyapunov function:

$$
\begin{aligned}
& V(P(t), L(t), S(t), R(t), Q(t)) \\
& \quad=P_{0}\left(\frac{P}{P_{0}}-\ln \frac{P}{P_{0}}\right)+L+S+R+Q .
\end{aligned}
$$

The derivative of $V$ is given by

$$
\begin{aligned}
V^{\prime}= & P^{\prime}-\frac{P_{0}}{P} P^{\prime}+L^{\prime}+S^{\prime}+R^{\prime}+Q^{\prime} \\
= & b-\beta_{1} P L-\left(d_{1}+\mu\right) P-\frac{b /\left(d_{1}+\mu\right)}{P} \\
& \times\left(b-\beta_{1} P L-\left(d_{1}+\mu\right) P\right)+\beta_{1} P L-\beta_{2} L S \\
& +\rho_{2} L R-\left(d_{2}+\mu\right) L+\beta_{2} L S+\rho_{1} S R \\
& -\left(\omega+d_{3}+\mu\right) S+\omega S-\rho_{1} S R \\
& -\rho_{2} L R-\left(\gamma+d_{4}+\mu\right) R+\gamma R-\left(d_{5}+\mu\right) Q \\
= & 2 b-\frac{b^{2}}{\left(d_{1}+\mu\right) P}-\left(d_{1}+\mu\right) P+\frac{\beta_{1} b}{d_{1}+\mu} L
\end{aligned}
$$

$$
\begin{aligned}
& -\left(d_{2}+\mu\right) L-\left(d_{3}+\mu\right) S-\left(d_{4}+\mu\right) R-\left(d_{5}+\mu\right) Q \\
= & {\left[2-\frac{b}{\left(d_{1}+\mu\right) P}-\frac{\left(d_{1}+\mu\right) P}{b}\right] b } \\
& +\left(\frac{\beta_{1} b}{d_{1}+\mu}-d_{2}-\mu\right) L-\left(d_{3}+\mu\right) S \\
& -\left(d_{4}+\mu\right) R-\left(d_{5}+\mu\right) Q \\
= & {\left[2-\frac{b}{\left(d_{1}+\mu\right) P}-\frac{\left(d_{1}+\mu\right) P}{b}\right] b } \\
& +\frac{\beta_{1} b-\left(d_{1}+\mu\right)\left(d_{2}+\mu\right)}{d_{1}+\mu} L-\left(d_{3}+\mu\right) S-\left(d_{4}+\mu\right) R \\
& -\left(d_{5}+\mu\right) Q .
\end{aligned}
$$

If $R_{0} \leq 1$, then $\beta_{1} b \leq\left(d_{1}+\mu\right)\left(d_{2}+\mu\right)$, so we get $\left(\beta_{1} b-\left(d_{1}+\right.\right.$ $\left.\mu)\left(d_{2}+\mu\right) /\left(d_{1}+\mu\right)\right) L \leq 0$.

As we know, $2-b /\left(d_{1}+\mu\right) P-\left(d_{1}+\mu\right) P / b \leq 0$, so we obtain $V^{\prime} \leq 0$ with equality only if $2-b /\left(d_{1}+\mu\right) P-\left(d_{1}+\right.$ $\mu) P / b=0, R_{0}=1$ and $S=R=Q=0$. By LaSalle invariance principle $[20,22], E_{0}$ is globally asymptotically stable. Thus, for system (2), the smoking-free equilibrium $E_{0}$ is globally asymptotically stable if $R_{0} \leq 1$.

3.2.2. Stability of the Occasion Smoking Equilibrium. In this part, we will consider an occasional smoking equilibrium $E_{L}\left(\left(d_{2}+\mu\right) / \beta_{1}, b /\left(d_{2}+\mu\right)-\left(d_{1}+\mu\right) / \beta_{1}, 0,0,0\right)$; that is, only potential smokers and occasionally smokers are not zero, and the other compartments are zero.

Theorem 5. If $R_{0}>1$, the occasion smoking equilibrium $E_{L}$ is locally asymptotically stable.

Proof. The Jacobian matrix of the giving up smoking model (2) around $E_{L}$ is given by

$$
J_{E_{L}}=\left(\begin{array}{cc}
-\frac{\beta_{1} b}{d_{2}+\mu} & -d_{2}-\mu \\
\frac{\beta_{1} b}{d_{2}+\mu}-\left(d_{1}+\mu\right) & 0
\end{array}\right) \text {. }
$$

The characteristic polynomial is $\psi(x)=x^{2}+c_{1} x+c_{2}$, where $c_{1}=\beta_{1} b /\left(d_{2}+\mu\right)$ and $c_{2}=\beta_{1} b-\left(d_{1}+\mu\right)\left(d_{2}+\mu\right)$. Therefore by Routh-Hurwitz criteria we deduce that the roots of the polynomial $\psi(x)$ have negative real part when $\beta_{1} b>$ $\left(d_{1}+\mu\right)\left(d_{2}+\mu\right)$, which shows that the system is locally asymptotically stable if $R_{0}>1$.

Theorem 6. If $R_{0}>1$ and $R_{1}=b \beta_{2} /\left(d_{2}+\mu\right)\left(d_{3}+\mu\right)-\left(d_{1}+\right.$ $\mu) \beta_{2} / \beta_{1}\left(d_{3}+\mu\right) \leq 1$, the occasion smoking equilibrium $E_{L}$ is globally asymptotically stable. 
Proof. We introduce the following Lyapunov function:

$$
\begin{aligned}
V(P(t), L(t), S(t), R(t), Q(t)) \\
=P_{L}\left(\frac{P}{P_{L}}-\ln \frac{P}{P_{L}}\right)+L\left(\frac{L}{L_{L}}-\ln \frac{L}{L_{L}}\right) \\
+S+R+Q .
\end{aligned}
$$

The derivative of $V$ is given by

$$
\begin{aligned}
V^{\prime}= & P^{\prime}-\frac{P_{L}}{P} P^{\prime}+L^{\prime}-\frac{L_{L}}{L} L^{\prime}+S^{\prime}+R^{\prime}+Q^{\prime} \\
= & 2 b-\frac{\left(d_{2}+\mu\right) b}{\beta_{1} P}-\frac{\beta_{1} P b}{d_{2}+\mu} \\
& +\left[\left(\frac{b}{d_{2}+\mu}-\frac{d_{1}+\mu}{\beta_{1}}\right) \beta_{2}-\left(d_{3}+\mu\right)\right] S \\
& -\left(\frac{b}{d_{2}+\mu}-\frac{d_{1}+\mu}{\beta_{1}}\right) \rho_{2} R \\
& -\left(d_{4}+\mu\right) R-\left(d_{5}+\mu\right) Q \\
= & \left(2-\frac{d_{2}+\mu}{\beta_{1} P}-\frac{\beta_{1} P}{d_{2}+\mu}\right) b \\
& +\left[\left(\frac{b}{d_{2}+\mu}-\frac{d_{1}+\mu}{\beta_{1}}\right) \beta_{2}-\left(d_{3}+\mu\right)\right] S \\
& -\left(\frac{b}{d_{2}+\mu}-\frac{d_{1}+\mu}{\beta_{1}}\right) \rho_{2} R \\
& -\left(d_{4}+\mu\right) R-\left(d_{5}+\mu\right) Q .
\end{aligned}
$$

If $R_{0}>1$, we can obtain $b /\left(d_{2}+\mu\right)-\left(d_{1}+\mu\right) / \beta_{1}>0$. As we know $2-\left(d_{2}+\mu\right) / \beta_{1} P-\beta_{1} P /\left(d_{2}+\mu\right) \leq 0$, if $\left(b /\left(d_{2}+\mu\right)-\right.$ $\left.\left(d_{1}+\mu\right) / \beta_{1}\right) \beta_{2} \leq d_{3}+\mu$, so we obtain $V^{\prime} \leq 0$ with equality only if $R_{1}=1$ and $R=Q=0$. By LaSalle invariance principle $[20,22], E_{L}$ is globally asymptotically stable. This completes the proof.

\subsubsection{Stability of the Smoking-Present Equilibrium}

Theorem 7. Under the condition (3) of the Theorem 3, if $S / S^{*}, R / R^{*}, Q / Q^{*}$ satisfy one of four relations as follows:

$$
\begin{gathered}
\frac{R}{R^{*}}<1, \quad \frac{Q}{Q^{*}}<1, \quad \frac{S}{S^{*}} \geq \frac{R}{R^{*}} \geq \frac{Q}{Q^{*}}, \\
\frac{R}{R^{*}}>1, \quad \frac{Q}{Q^{*}}>1, \quad \frac{S}{S^{*}} \leq \frac{R}{R^{*}} \leq \frac{Q}{Q^{*}}, \\
\frac{R}{R^{*}}>1, \quad \frac{Q}{Q^{*}}<1, \quad \frac{S}{S^{*}} \leq \frac{R}{R^{*}}, \quad \frac{R}{R^{*}} \geq \frac{Q}{Q^{*}}, \\
\frac{R}{R^{*}}<1, \quad \frac{Q}{Q^{*}}>1, \quad \frac{R}{S^{*}} \geq \frac{R}{R^{*}}, \quad \frac{R}{R^{*}} \leq \frac{Q}{Q^{*}} .
\end{gathered}
$$

Then smoking-present equilibrium $E^{*}\left(P^{*}, L^{*}, S^{*}, R^{*}, Q^{*}\right)$ is globally asymptotically stable.
Proof. At the equilibrium point, the expressions on the righthand side of system (2) give us following relations:

$$
\begin{aligned}
\left(d_{1}+\mu\right) & =\frac{b}{P^{*}}-\beta_{1} L^{*}, \\
\left(d_{2}+\mu\right) & =\beta_{1} P^{*}-\beta_{2} S^{*}+\rho_{2} R^{*}, \\
\left(\omega+d_{3}+\mu\right) & =\beta_{2} L^{*}+\rho_{1} R^{*}, \\
\left(\gamma+d_{4}+\mu\right) & =\omega \frac{S^{*}}{R^{*}}-\rho_{1} S^{*}-\rho_{2} L^{*}, \\
\left(d_{5}+\mu\right) & =\gamma \frac{R^{*}}{Q^{*}} .
\end{aligned}
$$

We now consider a candidate Lyapunov function $V$ such that

$$
\begin{aligned}
V= & \left(P-P^{*}-P^{*} \ln \frac{P}{P^{*}}\right)+\left(L-L^{*}-L^{*} \ln \frac{L}{L^{*}}\right) \\
& +\left(S-S^{*}-S^{*} \ln \frac{S}{S^{*}}\right)+\left(R-R^{*}-R^{*} \ln \frac{R}{R^{*}}\right) \\
& +\left(Q-Q^{*}-Q^{*} \ln \frac{Q}{Q^{*}}\right) .
\end{aligned}
$$

Then $V>0$ and the derivative of $V$ are given by

$$
\begin{aligned}
V^{\prime}= & \left(1-\frac{P^{*}}{P}\right) P^{\prime}+\left(1-\frac{L^{*}}{L}\right) L^{\prime}+\left(1-\frac{S^{*}}{S}\right) S^{\prime} \\
& +\left(1-\frac{R^{*}}{R}\right) R^{\prime}+\left(1-\frac{Q^{*}}{Q}\right) Q^{\prime} \\
= & \left(1-\frac{P^{*}}{P}\right)\left[b-\beta_{1} L P-\left(d_{1}+\mu\right) P\right] \\
& +\left(1-\frac{L^{*}}{L}\right)\left[\beta_{1} L P-\beta_{2} L S+\rho_{2} R L-\left(d_{2}+\mu\right) L\right] \\
& +\left(1-\frac{S^{*}}{S}\right)\left[\beta_{2} L S+\rho_{1} R S-\left(\omega+d_{3}+\mu\right) S\right] \\
& +\left(1-\frac{R^{*}}{R}\right)\left[\omega S-\rho_{1} R S-\rho_{2} R L-\left(\gamma+d_{4}+\mu\right) R\right] \\
& +\left(1-\frac{Q^{*}}{Q}\right)\left[\gamma R-\left(d_{5}+\mu\right) Q\right] .
\end{aligned}
$$


Using the relations in (49) we have

$$
\begin{aligned}
V^{\prime}= & \left(1-\frac{P^{*}}{P}\right)\left[b-\beta_{1} L P-\frac{P^{*}}{P} b+\beta_{1} P L^{*}\right] \\
+ & \left(1-\frac{L^{*}}{L}\right)\left[\beta_{1} L P-\beta_{2} L S+\rho_{2} R L-\beta_{1} P^{*} L\right. \\
& \left.+\beta_{2} L S^{*}-\rho_{2} R^{*} L\right] \\
+ & \left(1-\frac{S^{*}}{S}\right)\left[\beta_{2} L S+\rho_{1} R S-\beta_{2} L^{*} S-\rho_{1} R^{*} S\right] \\
+ & \left(1-\frac{R^{*}}{R}\right)\left[\omega S-\rho_{1} R S-\rho_{2} R L-\omega \frac{R}{R^{*}} S^{*}\right. \\
+ & \left.+\rho_{1} R S^{*}+\rho_{2} R L^{*}\right] \\
+ & \left(1-\frac{Q^{*}}{Q}\right)\left[\gamma R-\gamma \frac{Q}{Q^{*}} R^{*}\right] .
\end{aligned}
$$

We consider the following variable substitutions by letting

$$
\begin{gathered}
\frac{P}{P^{*}}=x, \quad \frac{L}{L^{*}}=y, \quad \frac{S}{S^{*}}=z, \\
\frac{R}{R^{*}}=u, \quad \frac{Q}{Q^{*}}=v .
\end{gathered}
$$

The derivative of $V$ reduces to

$$
\begin{aligned}
& V^{\prime}=\left(1-\frac{1}{x}\right)\left(b-x y \beta_{1} P^{*} L^{*}-x b+x \beta_{1} P^{*} L^{*}\right) \\
&+\left(1-\frac{1}{y}\right)\left(x y \beta_{1} P^{*} L^{*}+y u \rho_{2} R^{*} L^{*}-y z \beta_{2} L^{*} S^{*}\right. \\
&\left.-y \beta_{1} P^{*} L^{*}+y \beta_{2} L^{*} S^{*}-y \rho_{2} R^{*} L^{*}\right) \\
&+\left(1-\frac{1}{z}\right)\left(y z \beta_{2} L^{*} S^{*}+z u \rho_{1} R^{*} S^{*}\right. \\
&+\left(1-\frac{1}{u}\right)\left(z \omega S^{*}-z u \rho_{1} R^{*} S^{*}-y u \rho_{2} R^{*} L^{*} S^{*}-z \rho_{1} R^{*} S^{*}\right) \\
&\left.-u \omega S^{*}+u \rho_{1} R^{*} S^{*}+u \rho_{2} R^{*} L^{*}\right) \\
&+\left(1-\frac{1}{v}\right)\left(u \gamma R^{*}-v \gamma R^{*}\right) \\
&=\left(2-x-\frac{1}{x}\right) b+\left[\left(1-\frac{1}{x}\right)(x-x y)\right. \\
&\left.+\left(1-\frac{1}{y}\right)(x y-y)\right] \beta_{1} P^{*} L^{*}
\end{aligned}
$$

$$
\begin{aligned}
& +\left[\left(1-\frac{1}{y}\right)(y u-y)+\left(1-\frac{1}{u}\right)(u-y u)\right] \rho_{2} R^{*} L^{*} \\
& +\left[\left(1-\frac{1}{y}\right)(y-y z)+\left(1-\frac{1}{z}\right)(y z-z)\right] \beta_{2} L^{*} S^{*} \\
& +\left[\left(1-\frac{1}{z}\right)(z u-z)+\left(1-\frac{1}{u}\right)(u-z u)\right] \rho_{1} R^{*} S^{*} \\
& +\left(1-\frac{1}{u}\right)(z-u) \omega S^{*} \\
& +\left(1-\frac{1}{v}\right)(u-v) \gamma R^{*} \\
& =\left(2-x-\frac{1}{x}\right) b+\left(1-\frac{1}{u}\right)(z-u) \omega S^{*} \\
& +\left(1-\frac{1}{v}\right)(u-v) \gamma R^{*}
\end{aligned}
$$

as we know that $(2-x-1 / x) \leq 0$, when $z, u, v$ satisfy one of four relations as follows:

$$
\begin{aligned}
& u<1, \quad v<1, \quad z \geq u \geq v, \\
& u>1, \quad v>1, \quad z \leq u \leq v, \\
& u>1, \quad v<1, \quad \quad z \leq u, \quad u \geq v, \\
& u<1, \quad v>1, \quad z \geq u, \quad u \leq v .
\end{aligned}
$$

This implies that $V^{\prime} \leq 0$ with equality only if $P=P^{*}$ and $S^{*} / S=R^{*} / R=Q^{*} / Q$, that is, $x=1, z=u=v$. By LaSalle invariance principle $[20,22], E^{*}$ is globally asymptotically stable. This completes the proof.

Remark 8. It is possible for condition (48) to fail, in which case the global stability of the interior equilibrium of system (2) has not been established. Figure 2, however, seems to support the idea that the interior equilibrium of system (2) is still globally asymptotically stable even in this case.

\section{Numerical Simulation}

In this section, some numerical results of system (2) are presented for supporting the analytic results obtained previously. Our data are taken from [18], we also consider the data from Statistical Yearbook of the World Health [23] and Report of the Global Tobacco Epidemic [24]. Now, we give the data in Table 2.

According to the survey, the world population over the age of 15 is about 5.5 billion; this population is recorded as potential smokers, the smoking rate was $34 \%$. Hence, we will consider 5.5 billion, 2.2 billion, 1.87 billion, 0.058 billion, and 0.01 billion as the initial values of the five compartments.

Using the data in Table 2 we can get images in Figure 2.

The data in Table 2 satisfy the condition (3) of Theorem 3; we can see that the smoking-present equilibrium $E^{*}$ is globally asymptotically stable. 


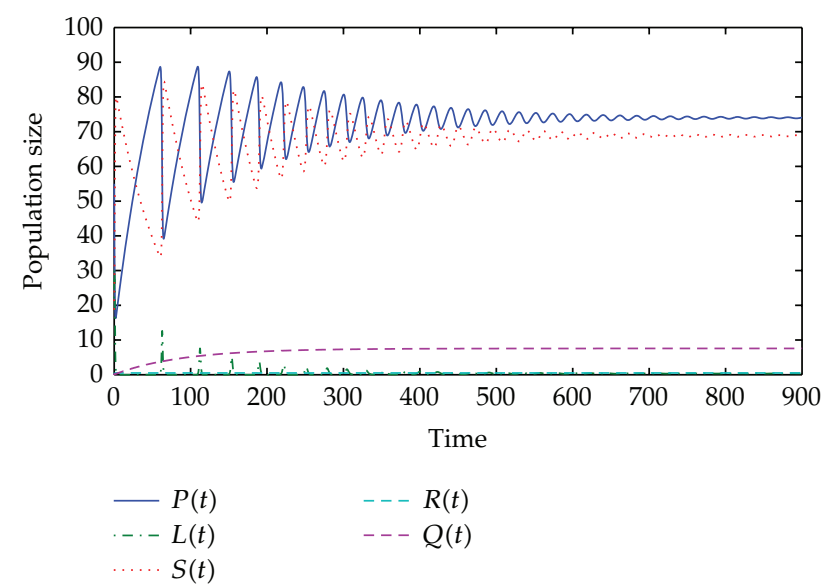

FIGURE 2: The smoking-present equilibrium $E^{*}$ is globally asymptotically stable.

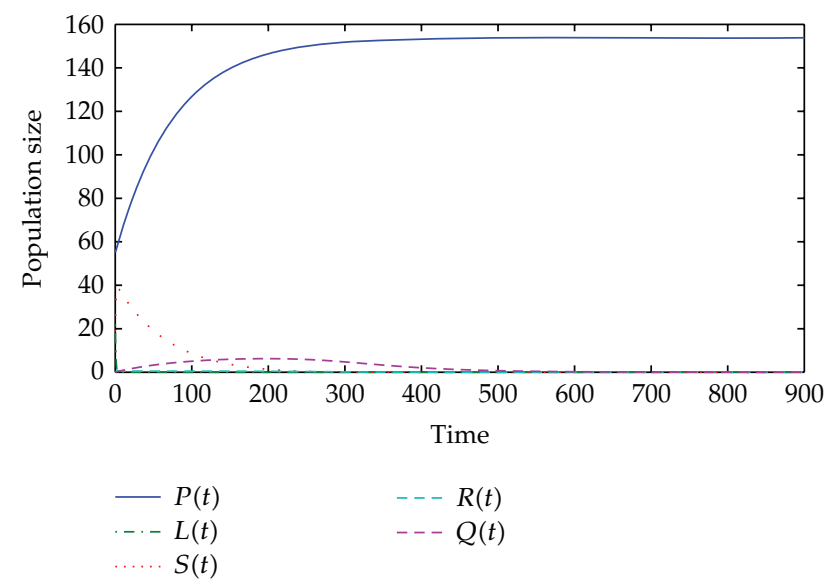

FIGURE 3: When $R_{0}<1$, the smoking-free equilibrium $E_{0}$ is globally asymptotically stable.

For appropriate adjustment parameters, we choose $\beta_{1}=$ 0.0038 , then the smoking-free equilibrium $E_{0}$ is globally asymptotically stable (Figure 3 ).

If we choose $\beta_{1}=0.4, \beta_{2}=0.5, \mu=0.1, d_{1}=0.1, d_{2}=$ $0.2, d_{3}=0.23$, numerical simulation gives $R_{0}>1$ and $R_{1}<1$; the occasion smoking equilibrium $E_{L}$ is globally asymptotically stable (Figure 4 ).

At last, we choose $\beta_{1}=0.000078125, b=0.02, \mu+d_{1}=$ $0.01, \mu+d_{2}=0.01$, numerical simulation gives $R_{0}=1$; then the smoking-free equilibrium $E_{0}$ is globally asymptotically stable (Figure 5).

\section{Discussion}

We have formulated a giving up smoking model with relapse and investigate their dynamical behaviors. By means of the next generation matrix, we obtain their basic reproduction number, $R_{0}$, which plays a crucial role. By constructing Lyapunov function, we prove the global stability of their equilibria: when the basic reproduction number is less than

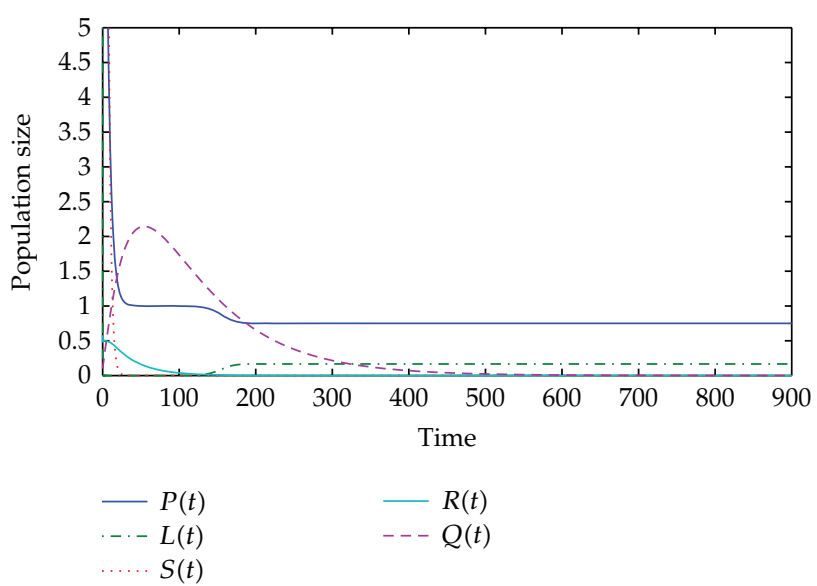

FIGURE 4: When $R_{0}>1$ and $R_{1}<1$, the occasion smoking equilibrium $E_{L}$ is globally asymptotically stable.

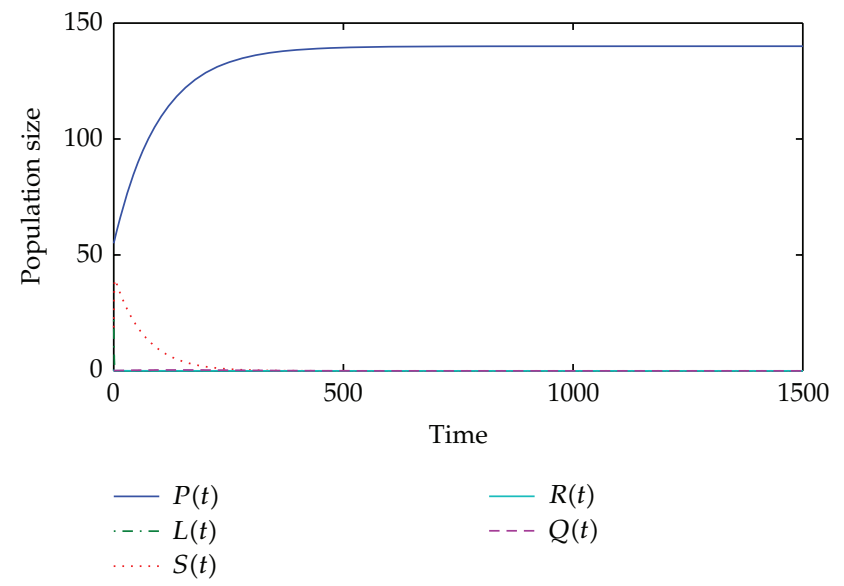

FIGURE 5: When $R_{0}=1$, the smoking-free equilibrium $E_{0}$ is globally asymptotically stable.

or equal to one, all solutions converge to the smoking-free equilibrium; that is, the smoking dies out eventually; when the basic reproduction number exceeds one, the occasion smoking equilibrium is stable; that is, the smoking will persist in the population, and the number of infected individuals tends to a positive constant.

In this paper, we consider two relapses. One is relapsed into light smokers and the other is relapsed into persistent smokers. If we employ some ways, such as medical care or education, to reduce the relapse rate, then, the number of the quit smokers will increase. We choose $\rho_{1}=\rho_{2}=0.003$, we can get images in Figure 6.

Comparison of Figures 2 and 6, we can see the difference between them. In Figure 6, the number of recovery and quit smokers is increasing obviously. $P(t), L(t), S(t), R(t)$ and $Q(t)$ are approaching the stable state earlier than the case of Figure 2 .

Through numerical simulation, we clearly recognize that if we control the rate of relapse, then the efficiency of giving up smoking will be greatly improved. 
TABLE 2: The parameter values of giving up smoking model.

\begin{tabular}{lcc}
\hline Parameter & Data estimated & Data sources \\
\hline$b$ & 0.2 year $^{-1}$ & Estimate \\
$\beta_{1}$ & 0.038 year $^{-1}$ & Estimate \\
$\beta_{2}$ & 0.0411 year $^{-1}$ & Estimate \\
$\rho_{1}$ & 0.081 year $^{-1}$ & Estimate \\
$\rho_{2}$ & 0.06 year $^{-1}$ & Estimate \\
$\omega$ & 0.041 year $^{-1}$ & Estimate \\
$\gamma$ & 0.0169 year $^{-1}$ & Estimate \\
$\mu$ & 0.0111 year $^{-1}$ & Reference [20] \\
$d_{1}$ & 0.0019 year $^{-1}$ & Reference [16] \\
$d_{2}$ & 0.0021 year $^{-1}$ & Reference [16] \\
$d_{3}$ & 0.0037 year $^{-1}$ & Reference [16] \\
$d_{4}$ & 0.0012 year $^{-1}$ & Reference [16] \\
$d_{5}$ & 0.0001 year $^{-1}$ & Estimate \\
\hline
\end{tabular}

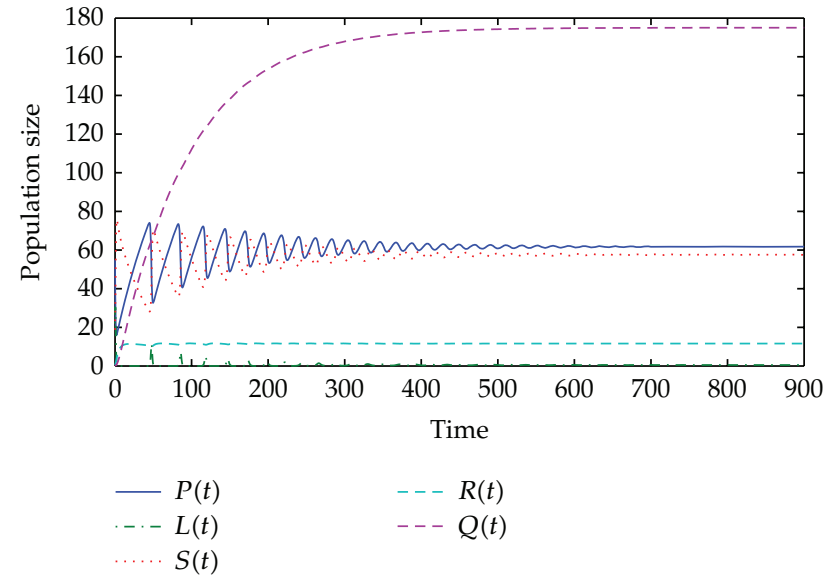

FIGURE 6: When $\rho_{1}=\rho_{2}=0.003$, the smoking-present equilibrium $E^{*}$ is globally asymptotically stable.

For system (2), $b$ reflects recruitment number, $\beta_{1}$ reflects the contact rate between potential smokers and occasion smokers, and $d_{1}$ denotes the deaths rate of potential smokers. $d_{2}$ denotes the deaths rate of light or occasion smokers. These four parameters will directly affect the values of the basic reproductive number. Furthermore, when $b$ and $\beta_{1}$ increase, the number smokers will increase, that is, $R_{0}$ increases. When we reduce the mortality caused by medical treatment, the number of permanent quit smokers will increase. Hence, $R_{0}$ will decrease. Figure 7 shows the relation between the basic reproduction number $R_{0}$ and $d_{1}$, Figure 8 shows the relation between the basic reproduction number $R_{0}$ and $d_{2}$, Figure 9 shows the relation between the basic reproduction number $R_{0}$ and $b$, Figure 10 shows the relation between the basic reproduction number $R_{0}$ and $\beta_{1}$. From Figures 11, 12, and 13 , we can also see that if $d_{1}$ and $d_{2}$ increase, then $R_{0}$ will decrease. And if $b$ and $\beta_{1}$ increase, then $R_{0}$ will increase. Biologically, this means that to reduce the relapse rate and the deaths rate of nicotine by medical treatment, education and legal constraints are very important.

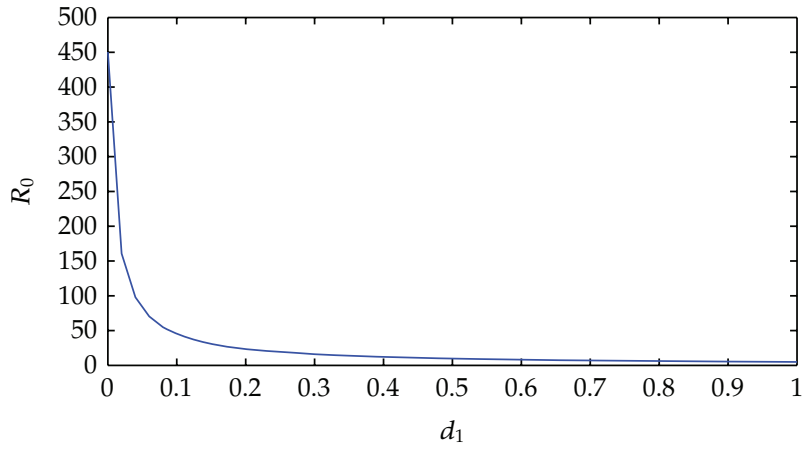

FIgURE 7: The relationship between $R_{0}$ and $d_{1}$.

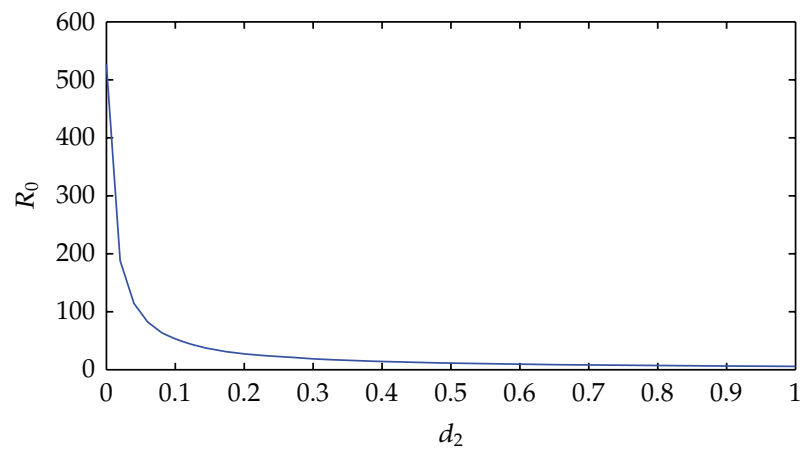

FIgURE 8: The relationship between $R_{0}$ and $d_{2}$.

Compared with [18], in this paper we add two bilinear relapse rates. Hence, our model is more closer to real life. In [18], the author only discussed the local asymptotic stability of occasional smoking equilibrium. In this paper, we give the proof of the global asymptotic stability of occasional smoking equilibrium, adding two bilinear relapse rates based on [18], our model becomes more complex. This brought difficulties to the discussion of the existence and stability of the endemic equilibrium. From (3) of Theorem 3, we know that if $R_{2}=1 / R_{0}+\left(d_{2}+\mu\right) \alpha_{3} / b \beta_{2}<1, d_{3} \rho_{2}-d_{2}<0$ 


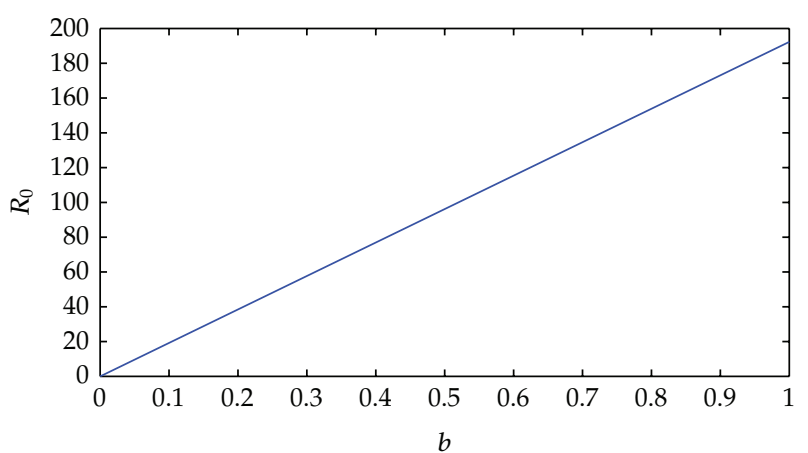

FIGURE 9: The relationship between $R_{0}$ and $b$.

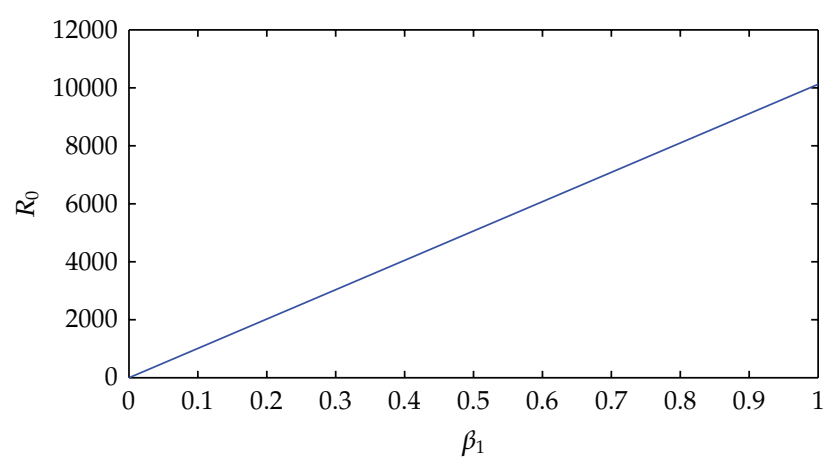

FIgURE 10: The relationship between $R_{0}$ and $\beta_{1}$.

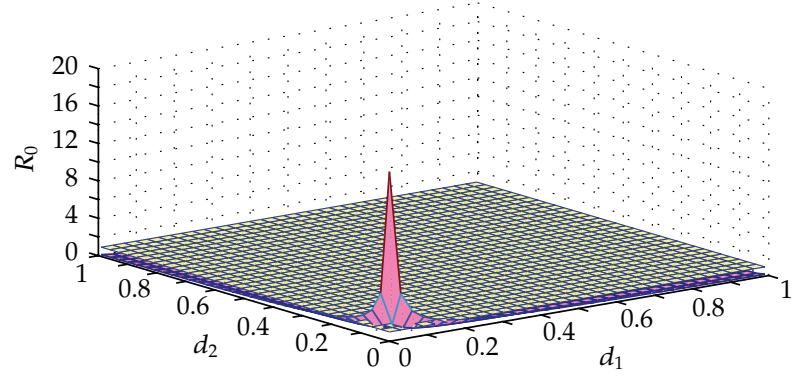

FIgURE 11: The relationship between $R_{0}, d_{1}$, and $d_{2}$.

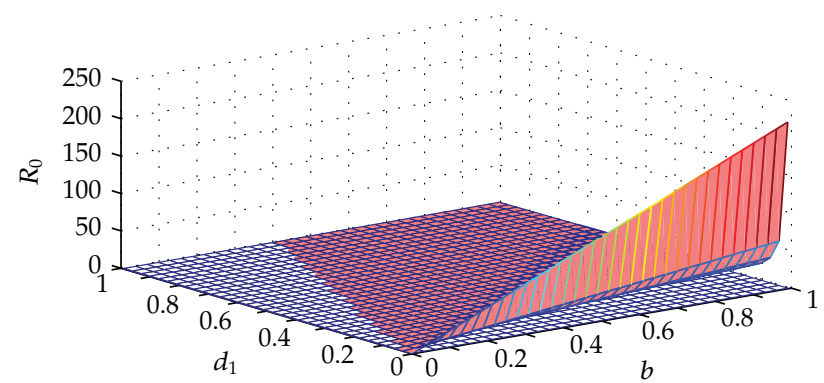

Figure 12: The relationship between $R_{0}, b$, and $d_{1}$.

and $\rho_{2}>\max \left\{d_{2} \rho_{1} \omega / d_{3} \alpha_{3}, \rho_{1} \omega / \alpha_{3}\right\}$, then system (2) has positive smoking-present equilibrium $E^{*}\left(P^{*}, L^{*}, S^{*}, R^{*}, Q^{*}\right)$ where $R^{*} \in\left(0, \omega / \rho_{1}\right)$. Hence, the numerical values of $\rho_{1}$ and $\rho_{2}$ are playing an important role in the existence of $E^{*}$. Next, we simulate the relationship between $\rho_{1}$ and $\rho_{2}$ under the conditions $R_{2}=1 / R_{0}+\left(d_{2}+\mu\right) \alpha_{3} / b \beta_{2}<1, d_{3} \rho_{2}-d_{2}<0$.

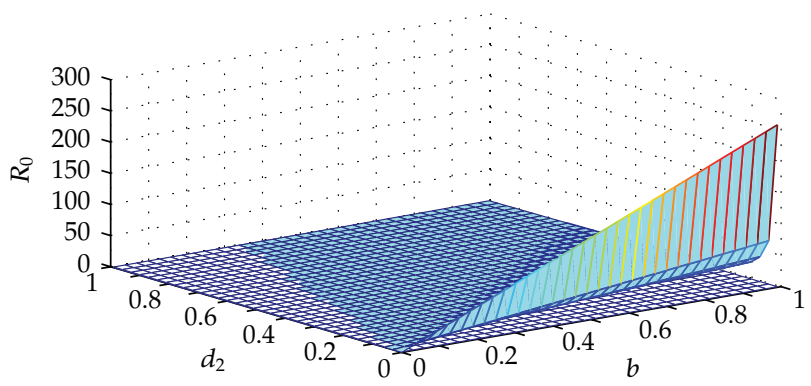

FIgURE 13: The relationship between $R_{0}, b$, and $d_{2}$.

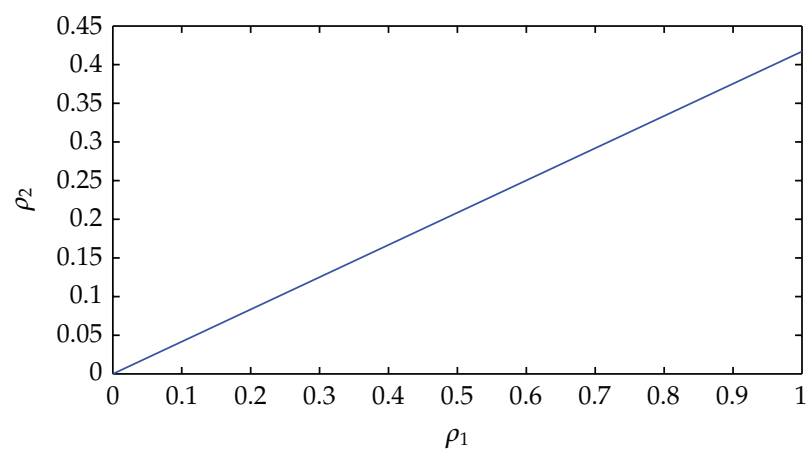

FIGURE 14: If $d_{2} \rho_{1} \omega / d_{3} \alpha_{3}>\rho_{1} \omega / \alpha_{3}$, the relationship between $\rho_{1}$ and $\rho_{2}$.

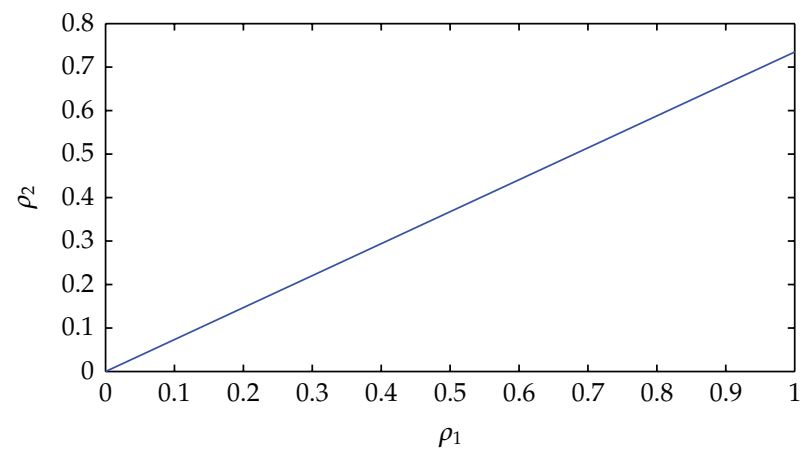

FIGURE 15: If $d_{2} \rho_{1} \omega / d_{3} \alpha_{3}<\rho_{1} \omega / \alpha_{3}$, the relationship between $\rho_{1}$ and $\rho_{2}$.

If $d_{2} \rho_{1} \omega / d_{3} \alpha_{3}>\rho_{1} \omega / \alpha_{3}$, we plot the relationship between $\rho_{1}$ and $\rho_{2}$ in Figure 14. If $d_{2} \rho_{1} \omega / d_{3} \alpha_{3}<\rho_{1} \omega / \alpha_{3}$, we plot the relationship between $\rho_{1}$ and $\rho_{2}$ in Figure 15. From Figures 14 and 15, we can know that the point which locates above the line is the positive smoking-present equilibrium.

\section{Acknowledgments}

This work was partially supported by the NNSF of China (10961018), the NSF of Gansu Province of China (1107RJZA088), the NSF for Distinguished Young Scholars of Gansu Province of China (1111RJDA003), the Special Fund for the Basic Requirements in the Research of University of Gansu Province of China, and the Development Program for 
HongLiu Distinguished Young Scholars in Lanzhou University of Technology.

\section{References}

[1] S. G. Ruan and W. D. Wang, "Dynamical behavior of an epidemic model with a nonlinear incidence rate," Journal of Differential Equations, vol. 188, no. 1, pp. 135-163, 2003.

[2] A. Korobeinikov, "Global properties of infectious disease models with nonlinear incidence," Bulletin of Mathematical Biology, vol. 69, no. 6, pp. 1871-1886, 2007.

[3] M. de la Sen and S. Alonso-Quesada, "Vaccination strategies based on feedback control techniques for a general SEIR epidemic model," Applied Mathematics and Computation, vol. 218, no. 7, pp. 3888-3904, 2011.

[4] M. de La Sen, R. P. Agarwal, A. Ibeas, and S. Alonso-Quesada, "On a generalized time-varying SEIR epidemic model with mixed point and distributed time-varying delays and combined regular and impulsive vaccination controls," Advances in Difference Equations, vol. 2010, Article ID 281612, 2010.

[5] A. M. Lyapunov, The General Problem of the Stability of Motion, Taylor \& Francis, London, UK, 1992.

[6] A. Korobeinikov, "Global properties of SIR and SEIR epidemic models with multiple parallel infectious stages," Bulletin of Mathematical Biology, vol. 71, no. 1, pp. 75-83, 2009.

[7] S. M. O’Regan, T. C. Kelly, A. Korobeinikov, M. J. A. O'Callaghan, and A. V. Pokrovskii, "Lyapunov function for SIR and SIRS epidmic models," Applied Mathematics Letters, vol. 23, pp. 446-448, 2010.

[8] B. Buonomo and S. Rionero, "On the Lyapunov stability for SIRS epidemic models with general nonlinear incidence rate," Applied Mathematics and Computation, vol. 217, no. 8, pp. 40104016, 2010.

[9] Z. H. Hu, P. Bi, W. B. Ma, and S. G. Ruan, "Bifurcations of an sirs epidemic model with nonlinear incidence rate," Discrete and Continuous Dynamical Systems B, vol. 15, no. 1, pp. 93-112, 2011.

[10] G. H. Li, W. D. Wang, and Z. Jin, "Global stability of an SEIR epidemic model with constant immigration," Chaos, Solitons and Fractals, vol. 30, no. 4, pp. 1012-1019, 2006.

[11] A. Korobeinikov, "Global properties of basic virus dynamics models," Bulletin of Mathematical Biology, vol. 66, no. 4, pp. 879-883, 2004.

[12] M. O. Souza and J. P. Zubelli, "Global stability for a class of virus models with cytotoxic T lymphocyte immune response and antigenic variation," Bulletin of Mathematical Biology, vol. 73, no. 3, pp. 609-625, 2011.

[13] H. F. Huo, S. J. Dang, and Y. N. Li, "Stability of a two-strain tuberculosis model with general contact rate," Abstract and Applied Analysis, vol. 2010, Article ID 293747, 31 pages, 2010.

[14] C. Castillo-Chavez and B. J. Song, "Dynamical models of tuberculosis and their applications," Mathematical Biosciences and Engineering, vol. 1, pp. 361-404, 2004.

[15] H. F. Huo and L. X. Feng, "Global stability for an HIV/AIDS epidemic model with different latent stages and treatment," Applied Mathematical Modelling, vol. 37, no. 3, pp. 1480-1489, 2013.

[16] R. Xu, "Global stability of an HIV-1 infection model with saturation infection and intracellular delay," Journal of Mathematical Analysis and Applications, vol. 375, no. 1, pp. 75-81, 2011.

[17] C. Castillo-Garsow, G. Jordan-Salivia, and A. RodriguezHerrera, "Mathematical models for the dynamics of tobacco use, recovery, and replase," Tech. Rep. BU-1505-M, Cornell University, 2000.

[18] G. Zaman, "Qualitative behavior of giving up smoking models," Bulletin of the Malaysian Mathematical Sciences Society, vol. 34, no. 2, pp. 403-415, 2011.

[19] G. Zaman, "Optimal campaign in the smoking dynamics, computation and mathematical methods in medicine," vol. 2011, Article ID 163834, 9 pages, 2011.

[20] R. Qesmi, J. Wu, J. H. Wu, and J. M. Heffernan, "Influence of backward bifurcation in a model of hepatitis B and C viruses," Mathematical Biosciences, vol. 224, no. 2, pp. 118-125, 2010.

[21] P. van den Driessche and J. Watmough, "Reproduction numbers and sub-threshold endemic equilibria for compartmental models of disease transmission," Mathematical Biosciences, vol. 180, pp. 29-48, 2002.

[22] J. P. LaSalle, The Stability of Dynamical Systems, Regional Conference Series in Applied Mathematics, SIAM, Philadelphia, Pa, USA, 1976.

[23] World Health Statistics, 2010, http://www.who.int/.

[24] WHO Report of the Global Tobacco Epidemic, 2011, http://www.who.int/. 


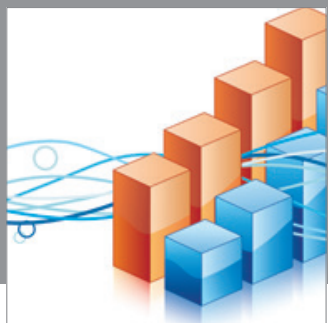

Advances in

Operations Research

mansans

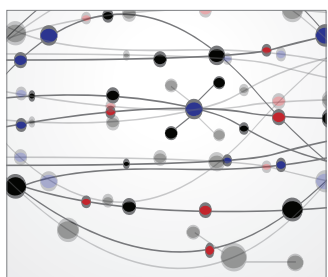

The Scientific World Journal
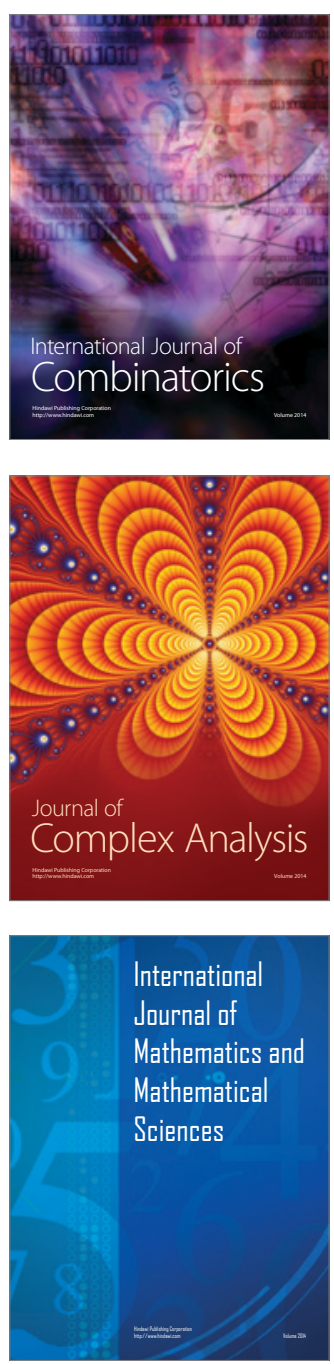
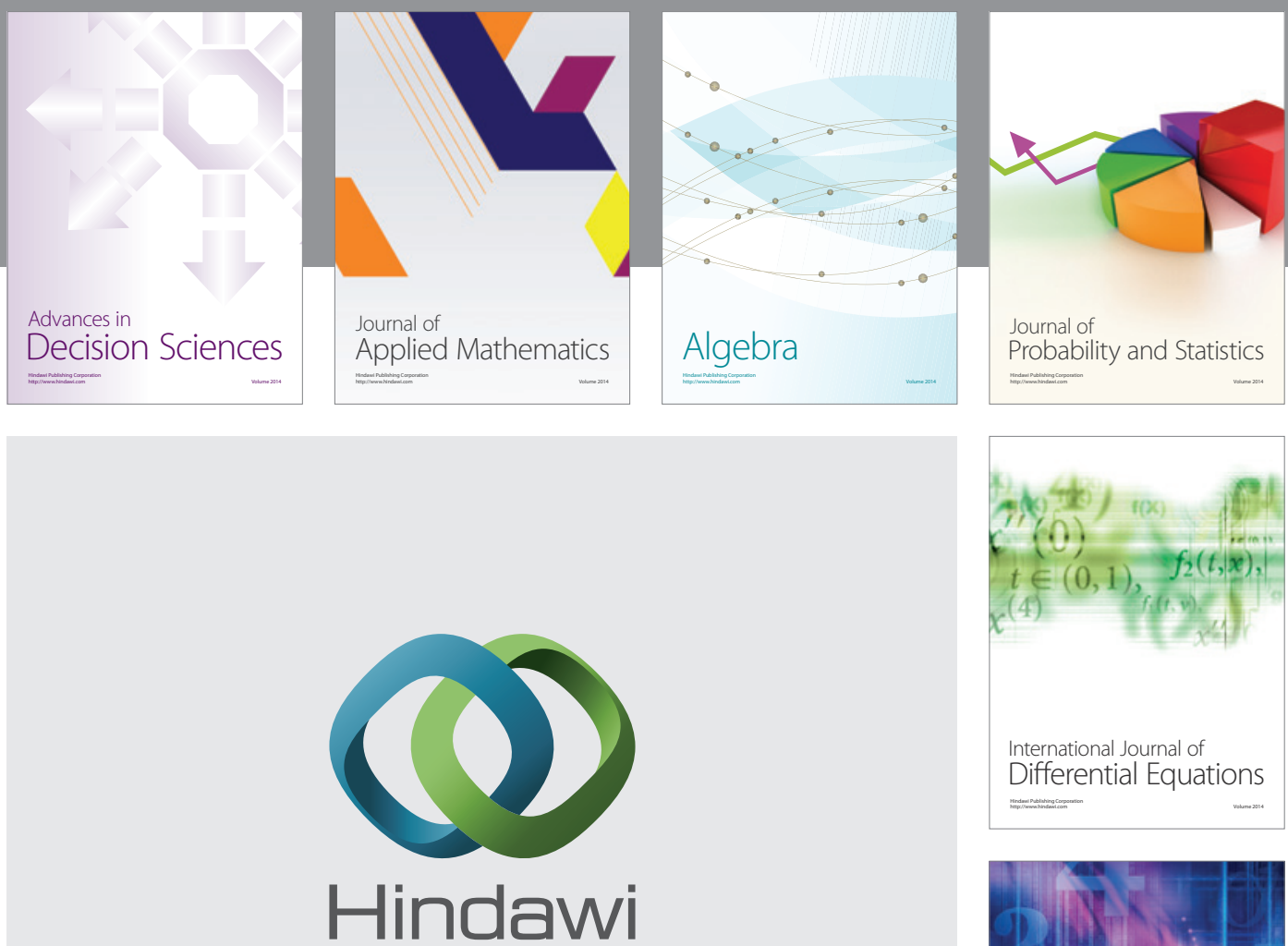

Submit your manuscripts at http://www.hindawi.com
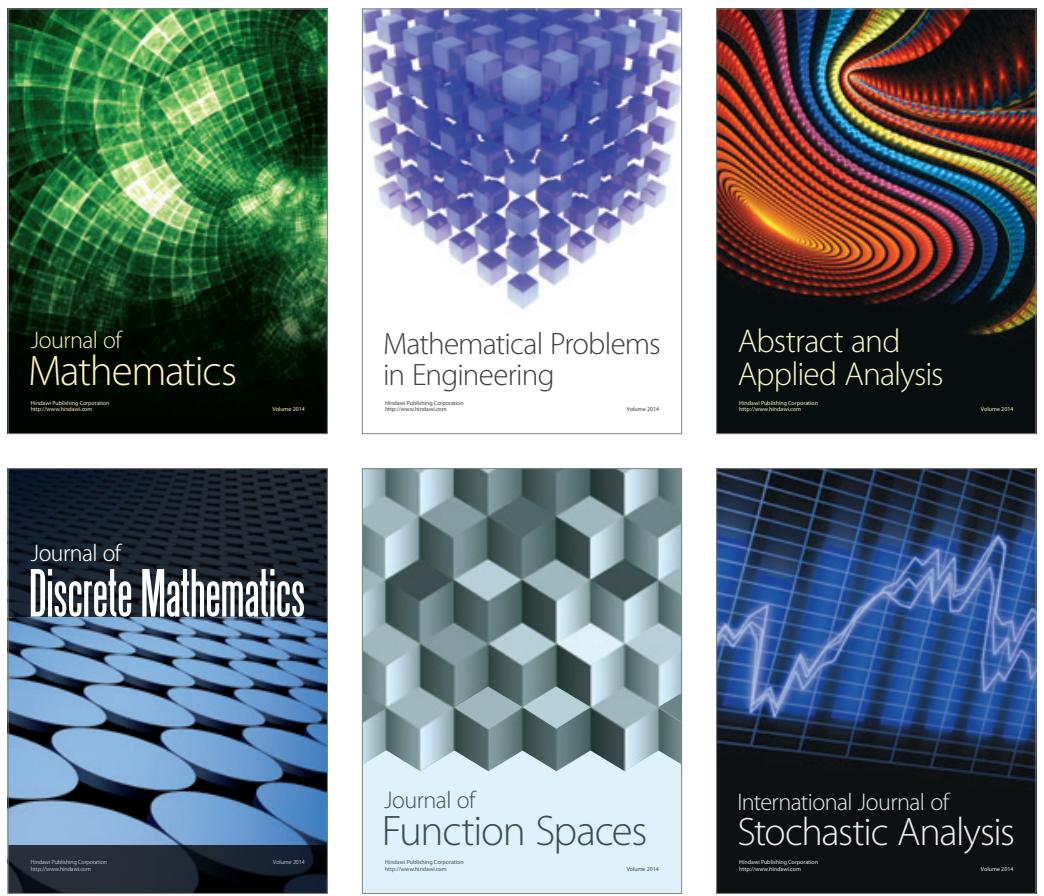

Journal of

Function Spaces

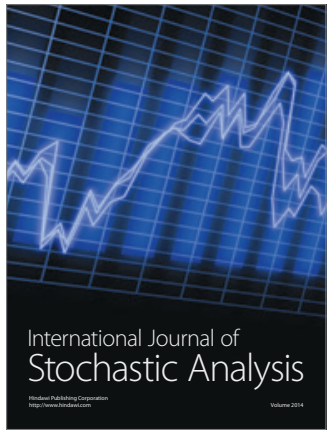

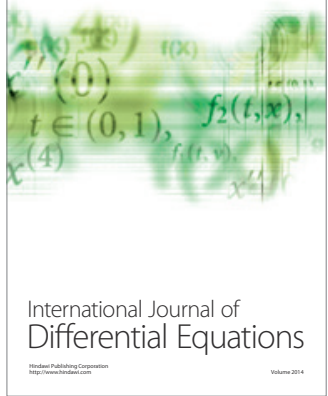
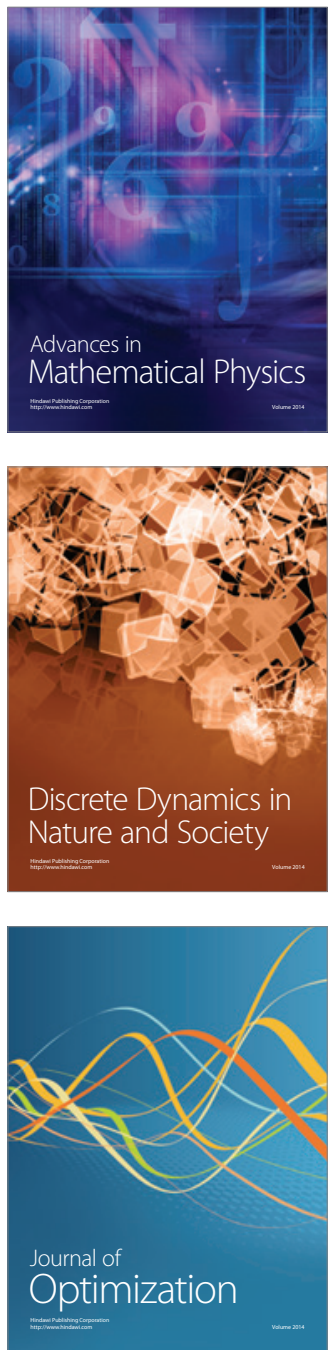